\title{
An Improved Ant Colony Matching by Using Discrete Curve Evolution
}

\author{
Younes Saadi ${ }^{1}$, Eka Novita Sari ${ }^{2}$, and Tutut Herawan ${ }^{1}$ \\ ${ }^{1}$ Department of Information System \\ University of Malaya \\ 50603 Pantai Valley, Kuala Lumpur, Malaysia \\ ${ }^{2}$ AMCS Research Center \\ Yogyakarta, Indonesia \\ younessaadi@gmail.com, eka@amcs.co, tutut@um.edu.my
}

\begin{abstract}
In this paper we present an improved Ant Colony Optimization (ACO) for contour matching, which can be used to match 2D shapes. Discrete Curve Evolution (DCE) technique is used to simplify the extracted contour. In order to find the best correspondence between shapes, the match process is formulated as a Quadratic Assignment Problem (QAP) and resolved by using Ant Colony Optimization (ACO). The experimental results justify that Discrete Curve Evolution (DCE) performs better than the previous Constant Sampling (CS) technique which has been selected for the ACO matching.
\end{abstract}

Keywords: Ant Colony Optimization, Quadratic assignment problem, Discrete curve evolution, Contour matching.

\section{Introduction}

Nowadays, shape analysis has become an important topic in computer vision. It has been widely adopted in many applications of computer graphics. In particular, shape matching one of the fundamental techniques of shape analysis. It plays a primordial role in shape retrieval, recognition and classification, and medical registration [1]. Shape matching has reached a state of maturity in which many real products based on shape matching are commercialized in different areas [2]. In the commercial domain, shape matching methods are being used to retrieve and classify images, for personal and institutional needs like security and military. In the medical domain, shape matching is used in radiology to diagnose and to assess medical images to determine the progress and the suitable treatment options.

Shape matching is application dependent. Different applications may have different requirements on invariance and tolerance to noise, distortion, blur, transformation, scale and orientation. Thus, it is difficult to design a universal method which is suitable for all applications. Nowadays, many techniques have been proposed but most of them only focus on the applications where shape is invariant to transformation. 
Based on the representation techniques, shape matching techniques can be classified into two categories: contour based matching and region based matching [3]. This research is focusing on contour based matching techniques, in which only the information located on the shape contour is explored. Majority of the past researches have concentrated on how to achieve a meaningful correspondence. On the other hand, some researches focused on improving representation methods of a contour in such a way that the resulted contour could be used to extract meaningful information. This is called simplification, evolution or smoothing in some sources [3] [4] [5] [6] [7].

However, challenges still remain. The first challenge is the invariance [8] [9]. Since shape in many applications is often discussed based on the property of invariance, shape matching is expected to be invariant to transformations such as example translation, scale and orientation. The second challenge is tolerance as noise, blur, crack and deformation are usually introduced when the shape of an object is extracted from an image [10]. In this case, a shape matching is required to be robust to these imperfections.

Moreover, in contour based matching, the points along the contours as a bipartite graph can be figured out and formulated as a QA. This is considered as an NP-hard problem [11]. In order to find an acceptable solution, heuristic1s techniques are often used. The main idea is to compute the mapping between two contours by minimizing the global dissimilarity. Many research studies have been proposed. For example, Hungarian method uses the simple greedy matching [12], and COPAP [13] takes into account the order preserving. However, the main drawback of these techniques is the omission of proximity information measurement between feature points on the same shape contour. For this reason, Kaick [14] proposed an Ant Colony Optimization (ACO) approach based on incorporating proximity into an optimization framework. However, the huge number of points incorporated in the correspondence makes the matching more complex and less accurate [11]. A modified ACO matching approach has been proposed by Ruberto and Morgera [11] based on genetic algorithm; only dominant points are used instead of the sampling distribution of contour points, which improves the correspondence accuracy and reduces the complexity.

Generally, speaking a heuristic is a method that achieves good (but not necessarily optimal) results at low expense. However, which results are to be considered good heavily depends on the application at hand. Analysing contour matching we can figure out the points along the contour as a bipartite graph, which can be formulated as a Quadratic Assignment (QA). This is one of the NP-hard problems [15]. Finding an optimal solution for such problem seems difficult by using conventional methods. Heuristics methods are used to find an acceptable solution for such cases.

It is important to mention the Hungarian method as one of the well-studied methods in this context. In fact it is based on solving the complexity which is a part of the combinatorial optimization solved by using Iterative Closest Point (ICP) scheme [16]. Following the requirements of the applications, many versions of heuristics approaches have been proposed. A review about correspondence introduced in [17] shows clearly the importance of heuristic methods to solve complexity related to shape matching issues.

In this paper, an improved ACO matching approach based on Discrete Curve Evolution (DCE) was proposed. In order to reduce the number of contour points incorporated in the correspondence, a polygonal approximation proposed previously by 
Latecki and Lakamper [18] was selected to simplify the extracted contour. Mainly, it simplifies the contour by neglecting distortions while at the same time preserving the perceptual appearance at a level sufficient for object recognition. To test the effectiveness of our approach, an MPEG-7 subset described by Ruberto and Morgera [11] was used to test shape retrieval considering noise and distortions effect. The results were also compared with the previous work of ACO matching by Ruberto and Morgera [11].

\section{Proposed Method}

The structure of the proposed method is shown in (Fig. 1). It operates into four stages. In the first stage the contour is traced by using a basic Matlab function. In the second stage the extracted contours are approximated by using Discrete Curve Evolution (DCE) which is proposed previously by Latecki and Lakamper [18]. After that, Ant Colony Optimization proposed previously [14] is used to compute the correspondence in the third stage. Finally in the fourth stage, the Euclidean distance has been selected to compute the dissimilarity between shapes according to the resulted matrix of correspondence.

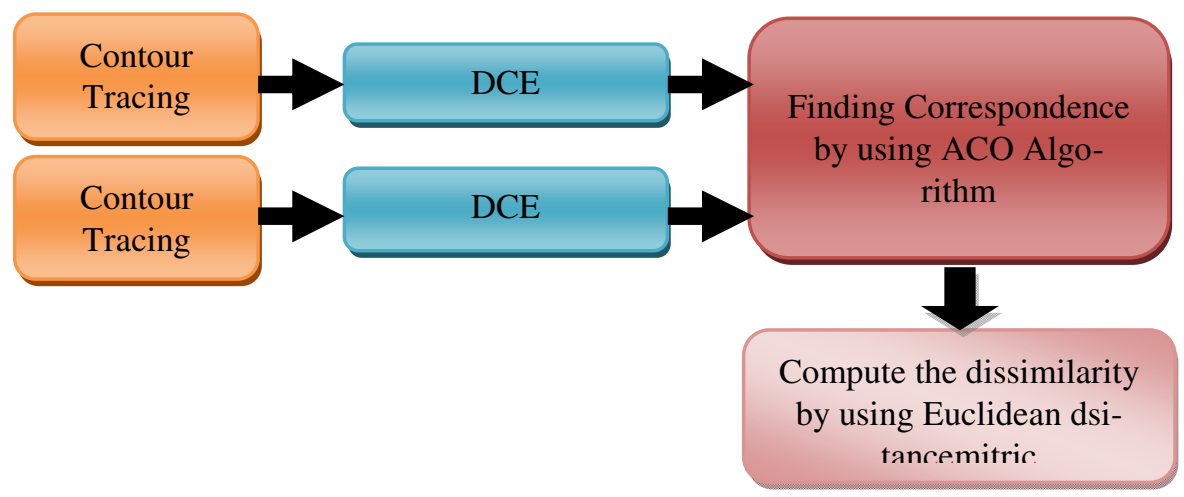

Fig. 1. Method structure

After analyzed the shortcoming of polygonal evolution methods, discrete curve evolution [18] has been selected to be used in our approach (See Figures 2, 3, and 4). The main reason is that this method allows the user to control the degree of evolution according to the human judgment. In every iteration, a pair of consecutive line segments $\mathrm{s} 1, \mathrm{~s} 2$ is replaced with a single line related to the endpoints of $\mathrm{s}_{1} \mathrm{U} \mathrm{s}_{2}$. The substitution is calculated according to the relevance value $K$ given by the following equation:

$$
\mathrm{K}\left(\mathrm{s}_{1}, \mathrm{~s}_{2}\right)=\frac{\beta\left(\mathrm{s}_{1}, \mathrm{~s}_{2}\right) \mathrm{l}\left(\mathrm{s}_{1}\right) \mathrm{l}\left(\mathrm{s}_{2}\right)}{\mathrm{l}\left(\mathrm{s}_{1}\right)+\mathrm{l}\left(\mathrm{s}_{2}\right)}
$$

$\beta\left(s_{1}, s_{2}\right)$ is the turn angle at the common vertex between $s_{1}$ and $s_{2} . l$ is the length function normalized with respect to the total length of a polygonal curve $\mathrm{C}$. 


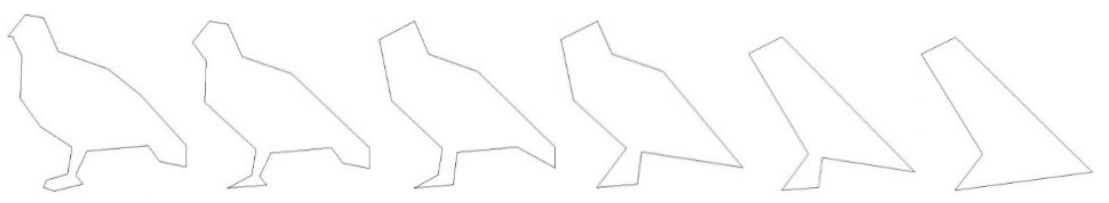

Fig. 2. A series of polygonal evolution

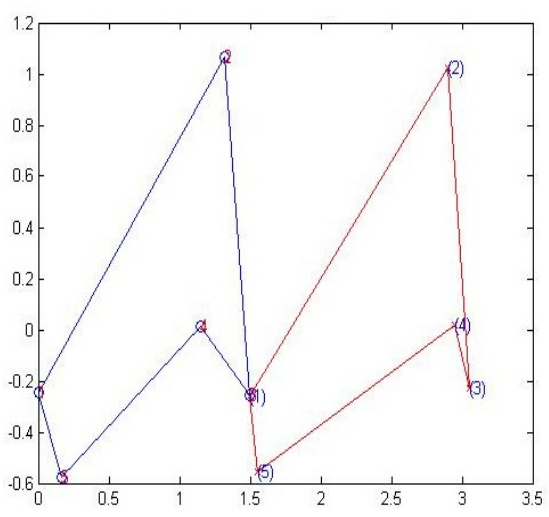

Fig. 3. Two simplified contours

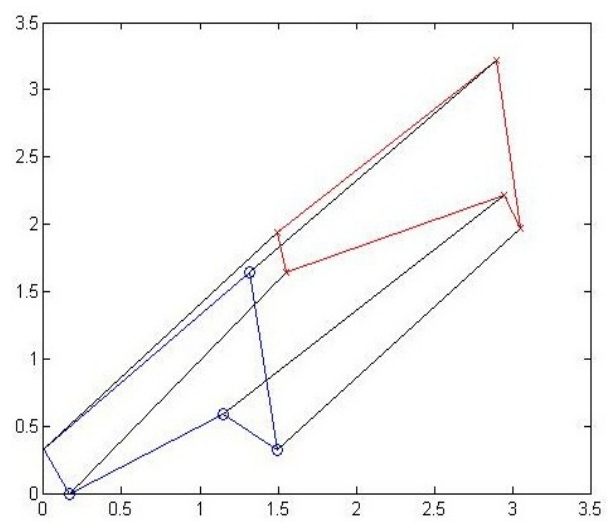

Fig. 4. ACO matching after DCE

A new matching algorithm based on ant colony optimization is described previously [14]. It consists of taking in consideration the proximity measured between feature points on the same shape (Fig. 5). The matching is formulated as a two sets of points I and $\mathrm{J}$ where the ants cross these two sets doing a complete tour. All the movements produce a collection of possible paths between the two sets. During building of these paths Ants release the pheromone with different amounts for each possible path. A bigger amount of pheromone on a path means that it is more eligible in term of cost of correspondence. The traversing from a vertex $i \in I$ to a vertex $j \in J$ is given by the equation below [11].

\subsection{Edge Probability}

$$
p_{i j}^{k}=\frac{\propto \tau_{i j}+(1-\propto) \eta_{i j}}{\sum_{l \in N_{i}}\left[\propto \tau_{i l}+(1-\propto) \eta_{i l}\right]} .
$$

The pheromone accumulated on the edge $(i, j)$ is quantified by $\tau_{i j}, \eta_{i j}$ indicates the desirability (or probability) of traversing (i,j) based on heuristic information, $\mathrm{N}_{\mathrm{i}}=\{\mathrm{l} \in \mathrm{J}:(\mathrm{i}, \mathrm{l}) \in \mathrm{E}\}$ is the immediate neighbourhood of vertex $\mathrm{i}$. The parameter $0 \leq \propto \leq 1$ regulates the influence of pheromones over heuristic information. After a complete tour of the ants, an ACO iteration, the cost of solutions is computed as defined in second.

Pheromones are updated at the end of ACO iteration. First, pheromones are evaporated at $n$ constant pheromone rate [11] $\rho, 0 \leq \rho \leq 1$. 


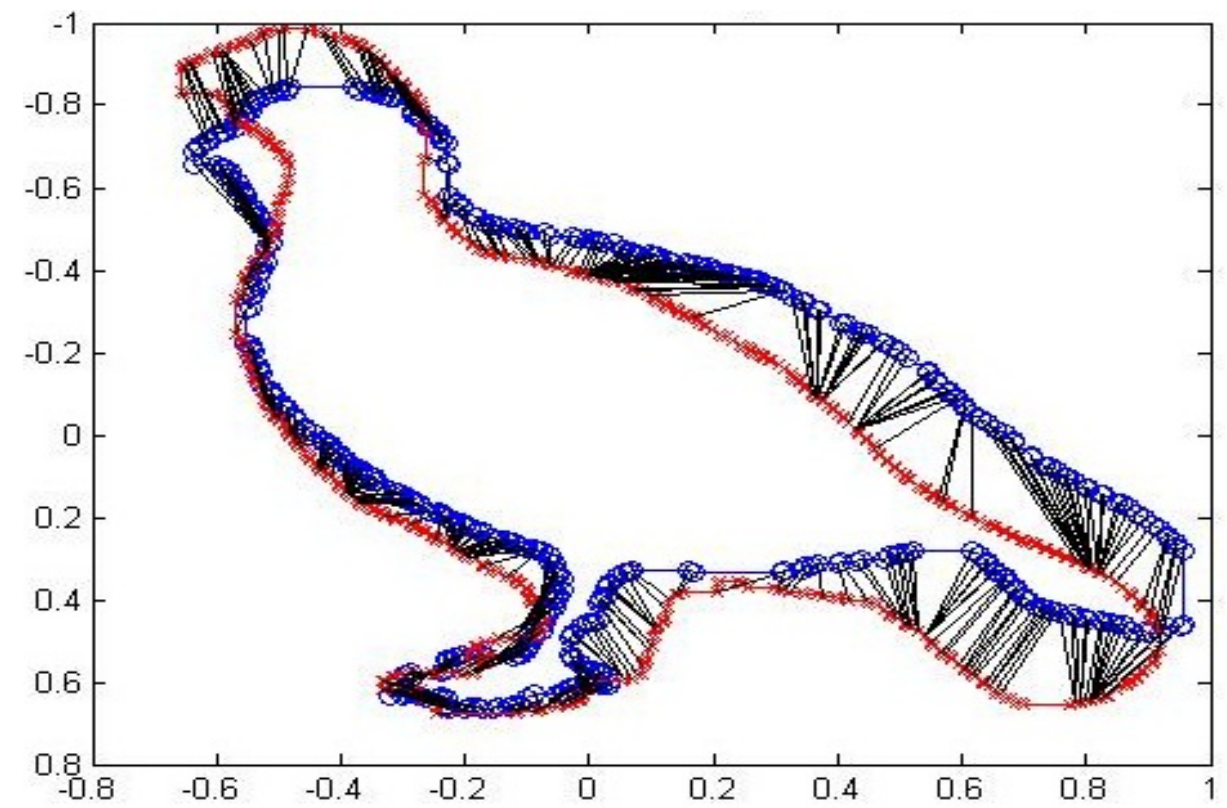

Fig. 5. ACO matching before DCE

\subsection{Pheromone Evaporation}

$$
\tau_{\mathrm{ij}} \leftarrow(1-\rho) \tau_{\mathrm{ij}},
$$

where $\rho$ is the pheromone evaporation rate and the new pheromone deposition on the edges that were traversed by the ants is regulated by pheromone deposition:

$$
\tau_{\mathrm{ij}} \leftarrow \tau_{\mathrm{ij}}+\sum_{\mathrm{k}=1}^{\mathrm{m}} \Delta \tau_{\mathrm{ij}}^{\mathrm{k}},
$$

where $\Delta \tau_{\mathrm{ij}}^{\mathrm{k}}$ is the amount of pheromone that an ant ${ }^{\mathbf{k}}$ has deposited on the edge $(\mathrm{i}, \mathrm{j})$.

\subsection{Cost Function}

The formulation of the problem is done by a QAP. When augmenting the shape descriptor $\mathrm{R}$ with proximity information. The general objective function is in the form:

$$
\mathrm{QAP}(\pi, \mathrm{R}, \mathrm{I}, J)=(1-v) \mathrm{S}(\pi, \mathrm{R}, \mathrm{I}, \mathrm{J})+v \chi(\pi, \mathrm{I}, \mathrm{J})
$$

where $0 \leq v \leq 1$ is used to control parameter between $\mathrm{S}$ and the proximity $\chi$ and the arguments I, J represents the two points sets, $\pi$ a mapping such that to a pint of I correspondents a point in $\mathrm{J}$ and $\mathrm{R}$ the set of shape descriptors. The form $\mathrm{S}$ and $\chi$ is detailed in [11]. 


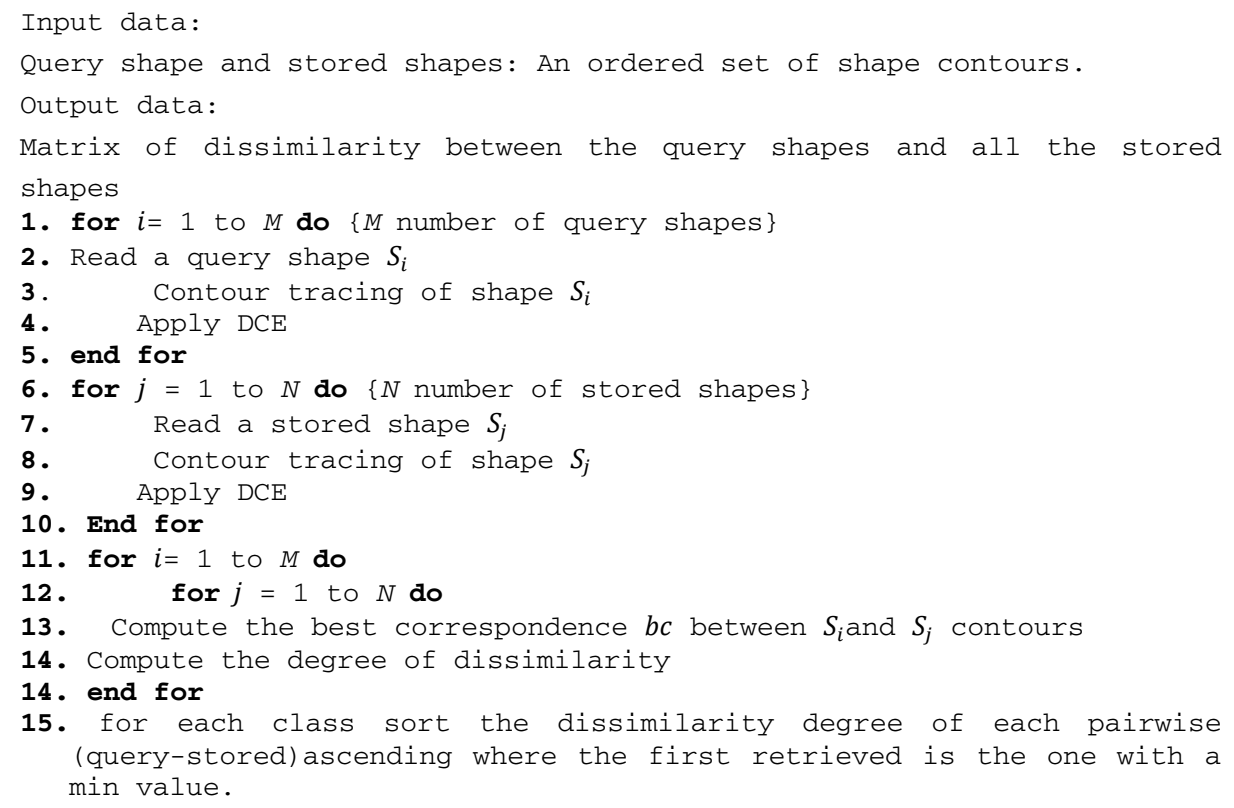

\section{Experimental Results}

In order to test the effectiveness of our approach, which is based on DCE and ACO matching, an MPEG-7 subset (Fig. 6) is used to test shape retrieval and contour matching issues [11]. The database constitutes of 18 classes, each class contains 11 items plus 18 query shapes selected from each class. Basically this database assesses universal measures that are translation rotation and scaling invariant. However, in our scope we are not interested in rotation since we are using the classic shape context as a shape descriptor. For this reason we are using a set of parameters consist of 1 as number of ants and 100 as number of iterations each ants make in optimization process. The experiment is executed on a Matlab environment (Windows 32 OS) running on Intel Pentium 2.2 GHz.

As stated in section 2, DCE reduce the number of points located on the contour. After the evolution, ACO is convoked to establish best possible correspondence between the approximated contours.

We compared our approach to retrieval similar objects by using Constant Sampling points (CS) [11]. It is important to mention that comparison is not possible with Dominant Points (DP) approach proposed by Ruberto and Morgera since it is based on rotational descriptor, which is not the case of our research. Table 1 shows the accuracy rate for each class of our method compared to the CS approach. In Table 1 we show the experiments made to MPEG-7 subset with CS and the proposed method. Table 1 also introduces a comparison with 18 classes for both CS and the proposed method. 


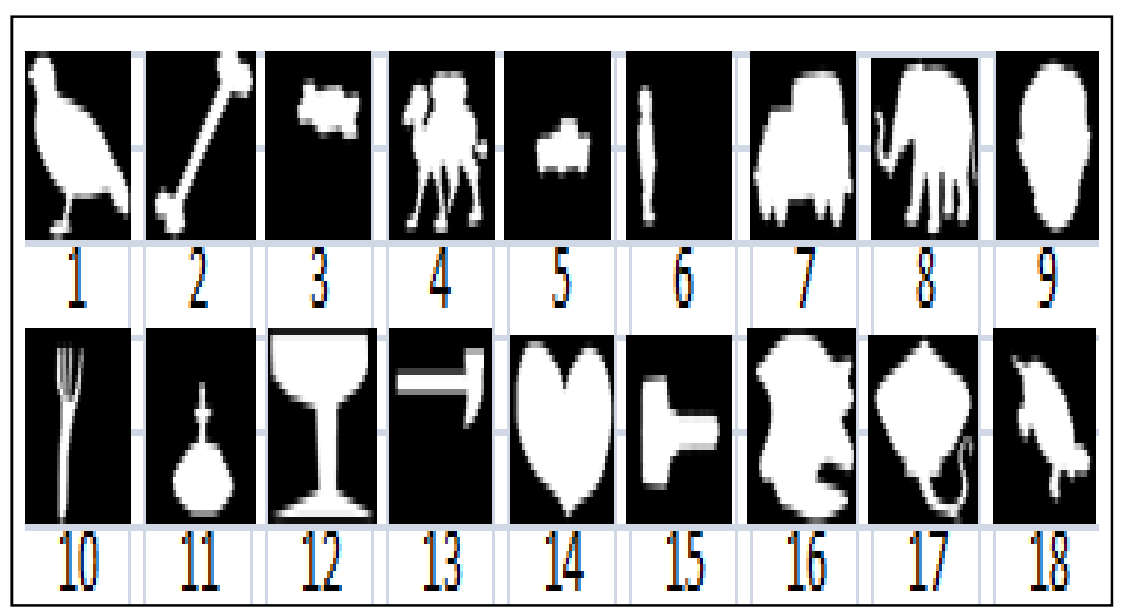

Fig. 6. MPEG-7 dataset [4]

Table 1. Retrieval rates of different MPEG-7 CE-Shape-1 Part B data set [11]

\begin{tabular}{|c|c|c|c|}
\hline & Class & $\begin{array}{c}\text { Proposed } \\
\text { Method }\end{array}$ & CS \\
\hline \multirow{18}{*}{ 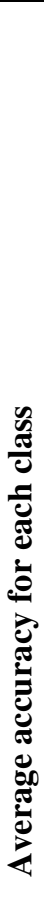 } & 1 & 0.9166 & 0.6909 \\
\hline & 2 & 1.0000 & 0.9273 \\
\hline & 3 & 0.9166 & 0.8818 \\
\hline & 4 & 0.8333 & 0.7273 \\
\hline & 5 & 0.9166 & 0.9909 \\
\hline & 6 & 1.0000 & 1.0000 \\
\hline & 7 & 0.9266 & 0.9909 \\
\hline & 8 & 0.9100 & 0.8818 \\
\hline & 9 & 1.0000 & 0.9727 \\
\hline & 10 & 1.0000 & 0.9455 \\
\hline & 11 & 0.9166 & 1.0000 \\
\hline & 12 & 1.0000 & 1.0000 \\
\hline & 13 & 0.5000 & 0.6818 \\
\hline & 14 & 1.0000 & 1.0000 \\
\hline & 15 & 0.9166 & 0.8545 \\
\hline & 16 & 1.0000 & 1.0000 \\
\hline & 17 & 0.5833 & 0.7545 \\
\hline & 18 & 0.6666 & 0.5636 \\
\hline Gl & Average & 0.8890 & 0.8854 \\
\hline
\end{tabular}


It is important to notice the highly performance affected by DCE compared to CS as a result of the lower number of points incorporated in the matching. There is dependence between lower accuracy and the smoothing of the contour. However th distribution of dominant points along the contour plays an important role to preserve the original frame of the shape and thus increasing the matching accuracy. The results obtained with classes 5 and 7 as shown in Table 1 by constant points CS is due to the nature of CS simplification.

Although the descriptor used in our approach is not invariant to rotation, the global average shows that our method performs better than CS. Thus the advantage of incorporating DCE and proximity using ACO is demonstrated.

\section{Conclusion}

In this study, an improved ACO matching algorithm based on curve evolution has been introduced. The main challenge to the contour matching was to find the best correspondence between the huge numbers of points along the contours incorporated in the matching. Whenever the number of points participated in the matching increased, the complexity of matching also increased which directly affected the performance of the matching process. A solution based on dominant points has been proposed by Ruberto and Morgera [11]. The obtained results showed the efficiency of our approach.

This research produced an improved ACO matching based on curve evolution. Before starting matching, a proposed DCE was applied in order to simplify the shape contour. This reduced the contour points incorporated along the contour. The DCE algorithmcan be personalized according to the number of iterations required. The iterations can be predefined to approximate the shape contour. Instead of the original ACO, the improved ACO matching as shown to be more accurate although the used descriptor is not invariant to rotation.

This does not mean, that heuristics methods might be useful for all matching applications, but the applicability of such methods is only related to: firstly, the domain of application for example sometimes in image registration it is enough to get an approximated matching to obtain the abnormalities. Secondly the applicability depends the requirements of the matching for example in some cases considering proximity between the points along the contour is compulsory which allow the usage of heuristic methods as a key for the solution.

Acknowledgments. This work is supported by University of Malaya High Impact Research Grant no vote UM.C/625/HIR/MOHE/SC/13/2 from Ministry of Higher Education Malaysia.

\section{References}

1. Tsapanos, N., Tefas, A., Nikolaidis, N., Pitas, I.: Shape matching using a binary search tree structure of weak classifiers. Pattern Recognition 45(6), 2363-2376 (2012)

2. Aaron, S.K., Christopher, R.I., Sharon, C.G.: Characterizing complex particle morphologies through shape matching: descriptors, applications, and algorithms. Journal of Computational Physics 230(17), 20 (2011) 
3. Luciano, D.F.C., Roberto, M.C.J.: Shape Classification and Analysis: Theory and Practice, 2nd edn. CRC Press (2009)

4. Kolesnikov, A.: ISE-bounded polygonal approximation of digital curves. Pattern Recognition Letters 33(10, 15), 1329-1337 (2012)

5. Parvez, M.T., Sabri, A.M.: Polygonal approximation of digital planar curves through adaptive optimizations. Pattern Recognition 31(13), 1997-2005 (2010)

6. Poyato, A.C., Cuevas, F.J.M., Carnicer, R.M., Salinas, R.M.: Polygonal approximation of digital planar curves through break point suppression. Pattern Recognition 43(1), 14-25 (2010)

7. Parvez, M.T., Sabri, A.M.: Polygonal Approximation of Planar Curves Using Triangular Suppression. In: Proceeding of International Conference on Information Science, Signal Processing and their Applications (2010)

8. Aaron, S.K., Christopher, R.I., Sharon, C.G.: Characterizing complex particle morphologies through shape matching: descriptors, applications, and algorithms. Journal of Computational Physics 230(17), 6438-6463 (2011)

9. de Sousa, S., Artner, N.M., Kropatsch, W.G.: On the Evaluation of Graph Centrality for Shape Matching. In: Kropatsch, W.G., Artner, N.M., Haxhimusa, Y., Jiang, X. (eds.) GbRPR 2013. LNCS, vol. 7877, pp. 204-213. Springer, Heidelberg (2013)

10. Li, G., Kim, H., Tan, J.K., Ishikawa, S.: 3D Organic Shape Correspondence Using Spherical Conformal Mapping. In: World Congress on Medical Physics and Biomedical Engineering, Beijing, China, May 26-31. IFMBE Proceedings, vol. 39, pp. 943-946 (2013)

11. Ruberto, C.D., Morgera, A.: ACO contour matching: a dominant point approach. In: 4th International Congress on Image and Signal Processing, vol. 03, pp. 1391-1395 (2012)

12. Papadimitriou, C., Stieglitz, K.: Combinatorial Optimization: Algorithms and Complexity. Prentice Hall (1982) ISBN:0-13-152462-3

13. Scott, C., Nowak, R.D.: Robust contour matching via the order-preserving assignment problem. IEEE Trans. on Image Processing 15(7), 1831-1838 (2006)

14. Kaick, O.V., Hammarneh, G., Zhang, H., Wighton, P.: Contour Correspondence Via Ant Colony Optimization. In: Proc. 15th Pacific Graphic 2007, United States, pp. 271-280 (2007)

15. Zhu, X.: Shape Matching Based on Ant Colony Optimization. In: Huang, D.-S., Wunsch II, D.C., Levine, D.S., Jo, K.-H. (eds.) ICIC 2008. CCIS, vol. 15, pp. 101-108. Springer, Heidelberg (2008)

16. Rusinkiewicz, S., Levoy, M.: Efficient Variants of the ICP Algorithm. In: Third International Conference on 3-D Digital Imaging and Modeling, pp. 145-152. IEEE (2001)

17. Kaick, O.V., Zhang, H., Hamarneh, G., Cohen-Or, D.: A survey on shape correspondence. Computer Graphics Forum 30(6), 1681-1707 (2011)

18. Latecki, L.J., Lakämper, R.: Shape Similarity Measure Based on correspondence of visual parts. IEEE Transactions on Pattern Analysis and Machine Intelligence 22(10), 1185-1190 (2000) 